\section{Reconstruction after partial duodenectomy using a Roux-en-Y lateral duodenoje- junostomy: a single center retrospective analysis}

\author{
Sumana Narayanan, ${ }^{1}$ Georg Herlitz, \\ Daniela Gomez, ${ }^{1}$ Laleh Melstrom, ${ }^{1,2}$ David
}

A. August, ${ }^{1,2}$ Darren R. Carpizo ${ }^{1,2}$

'Department of Surgery, Rutgers Robert Wood Johnson Medical School, New

Brunswick; ${ }^{2}$ Rutgers Cancer Institute of

New Jersey, New Brunswick, NJ, USA

\section{Abstract}

Oncologic resections of the second and third portions of the duodenum (D2 and D3) via partial duodenectomy can pose a challenging clinical problem. The duodenum must be repaired primarily or reconstructed. We have adopted a method of reconstruction using a Roux-en-Y duodenojejunostomy (D-J) in patients with extensive duodenal wall loss. We report our results in the first series of these cases. A retrospective review of patients who underwent post partial duodenectomy with reconstruction between June 2004 and March 2014 was performed. Five patients underwent partial duodenectomy with reconstruction. Two had resection for extrinsic tumors (colonic adenocarcinoma). Three had intrinsic duodenal tumors (one tubular adenoma, one adenocarcinoma and one gastrointestinal stromal tumor). All patients were reconstructed via retrocolic Roux-en-Y D-J. Mean estimated blood loss was $470 \mathrm{~mL}$ with median length of stay of 11 days. Post-operative complications included three intra-abdominal abscesses, one superficial wound infection and one gastrointestinal bleed. There were no anastomotic leaks, injuries to the ampulla of Vater or mortalities. In conclusion, partial duodenectomy of lateral D2/D3 with Roux-en-Y D-J is a relatively safe and effective alternative to partial duodenectomy with primary repair or pancreaticoduodenectomy for certain tumors of the duodenum.

\section{Introduction}

Oncologic resections for neoplasms involving the second and third portions of the duodenum (D2 and D3) can pose a challenging clinical problem because of the close proximity and fixation of the duodenum to the pancreas, the common opening of the common bile duct
(CBD) and major pancreatic duct at the ampulla of Vater as well as their shared blood supplythe superior and inferior pancreaticoduodenal arteries. ${ }^{1}$ For malignant neoplasms that require lymphadenectomy along with resection of the primary tumor such as duodenal adenocarcinomas, the optimal resection is a pancreaticoduodenectomy [(PD) or Whipple procedure]. However, for benign neoplasms intrinsic to the duodenum, extrinsic cancers (such as T4 colon cancer) or in malignant neoplasms that do not require a lymphadenectomy (i.e. gastrointestinal stromal tumors), the optimal method for surgical resection is not clear. The options include local excision of the duodenum which requires repair or pancreaticoduodenectomy.

Local excisions of the duodenum are an attractive option because the morbidity of a PD can be avoided with either a segmental duodenectomy or an excision of the lateral wall of the duodenum (partial duodenectomy). Moreover, choices to repair the defect and restore intestinal continuity vary from primary repair of the defect to reconstruction of the lateral wall using either a serosal patch, intestinal free flap or a Roux en-Y duodenojejunostomy (D-J). ${ }^{1-3}$ When greater than one third to one half of the circumference of the duodenum is resected, primary repair is not preferable due to the potential for luminal narrowing and leak at the suture line; making reconstruction methods more attractive. Studies reporting results of several of these options however are lacking. We have adopted a method of reconstruction using Roux-en-Y D-J which up to now has only been described in case reports. We sought here to report our results.

\section{Materials and Methods}

Five patients underwent partial duodenectomy with Roux-en-Y reconstruction between June 2004 and March 2014 by three oncologic surgeons. Clinical and operative data on these patients was compiled in a secure database which was retrospectively reviewed. Study approval was obtained from Rutgers University Institutional Review Board.

Data extracted from this database included patient demographics, co-morbidities [including American Society of Anesthesiologists (ASA) classification ], oncologic treatment histories and peri-operative nutritional status. Operative data such as method of resection and reconstruction, estimated blood loss (EBL), operative length, and transfusion data were recorded. Pathologic data including diagnosis and margin status as well as outcomes data including complication frequency, severity (by Clavien-Dindo classification) and length of stay were recorded.
Correspondence: Darren R. Carpizo, Rutgers Cancer Institute of New Jersey, 195 Little Albany St., New Brunswick, NJ 08903, USA.

Tel: +1.732.2358524 - Fax: +1.732.2358098.

E-mail: carpizdr@cinj.rutgers.edu

Key words: Duodenum; GIST; Adenoma; Roux-en-Y

Received for publication: 11 November 2014.

Revision received: 11 February 2015.

Accepted for publication: 12 February 2015.

This work is licensed under a Creative Commons Attribution NonCommercial 3.0 License (CC BYNC 3.0).

(C) Copyright S. Narayanan et al., 2015

Licensee PAGEPress, Italy

Surgical Techniques Development 2015; 5:5715 doi:10.4081/std.2015.5715

\section{Operative technique}

In all cases we began the operation by completing a Kocher maneuver to assess the relationship of the tumor to the duodenum for extrinsic tumors as well as to palpate the tumors that were intrinsic to the duodenum (Figure 1). One of the most important steps is estimating the location of the tumor (either intrinsic or extrinsic) in relation to the ampulla of Vater. If the proximity of the tumor to the ampulla was close, we often employed a maneuver in which we inserted a 4 French Fogarty catheter into the CBD and into the duodenum through the ampulla to facilitate localization of the ampulla. The bile duct was usually accessed via the cystic duct by performing a cholecystectomy. We then would make a lateral duodenotomy and with the ampulla localized, the decision was then made to excise the lateral wall of the duodenum or perform a segmental duodenal resection. In the case of duodenal adenomas, we sent the specimen for frozen section analysis to rule out an invasive cancer.

In these cases, reconstruction was performed using a Roux-en-Y limb of jejunum. The jejunum was divided and the Roux limb was brought to the duodenum in a retrocolic manner and anastomosed to the duodenum using a two-layer, hand sewn, side-to-side anastomosis. The proximal jejunum was than anastomosed to the Roux limb 60 to $70 \mathrm{~cm}$ distal to the D-J.

\section{Results}

From June 2004 to March 2014 five patients underwent partial resection of the duodenal wall with Roux-en-Y reconstruction. The 
demographics, co-morbidities, nutritional status and chemotherapy histories are listed in Table 1. Of these patients three were male and two were female with a mean age of 62 years (range 49-70 years old) and a mean body mass index of 27.9. Overall the patients were relatively mal-nourished with a mean preoperative albumin of 3.4 (range 2.8-4.2). Some of these patients also had significant comorbidities including one with cardiac disease, three with hypertension, one diabetic and one patient with chronic obstructive pulmonary disease. The median ASA classification was three.

Of the five patients, two had resection for tumors extrinsic to the duodenum (colonic adenocarcinoma), and three had resections of tumors intrinsic to the duodenum, including patients with a duodenal adenoma, duodenal adenocarcinoma and a gastrointestinal stromal tumor (GIST). Further details regarding the indications and extent of the operations are in the following sub-paragraphs.

\section{Patient 1}

A 67-year-old man presented with melena and was found on endoscopy to have a $1.2 \mathrm{~cm}$ moderately to poorly differentiated adenocarcinoma of the duodenum. The patient was taken to the operating room in anticipation of performing a Whipple procedure. However, intraoperatively the tumor was found on the duodenal free wall of distal D2. Palpation of the tumor suggested that it could be adequately removed via lateral duodenectomy. A partial duodenal wall resection encompassing the tumor was performed involving $2 / 3$ of the cir- cumference of the duodenal wall with negative margins on frozen section and a T1b pathologic stage. Due to the low stage of the tumor without nodal involvement and negative pathologic margins upon resection we felt from an oncologic perspective that we could proceed with reconstruction of the duodenal wall. This was able to be accomplished after the large duodenal wall resection by ensuring that the ampulla/CBD was not injured. This was done by making a choledochotomy in the cystic duct and threading a Fogarty balloon down to the ampulla of Vater confirming that it was intact, thus enabling us to safely proceed with the DJ. A T-tube was secured within the choledochotomy. The patient's hospital course was complicated by development of perihepatic abscess drained by interventional radiology and treated with antibiotics as well as a biliary leak from around the T-tube discovered on post-operative day 14 which required drainage of a second fluid collection. Ultimately the patient was followed for seven years post-operatively with no recurrence of disease.

\section{Patient 2}

A 49-year-old man with synchronous metastatic colon cancer to the liver with a large bulky primary tumor in the transverse colon and extension into lateral D2 and D3 portions of the duodenum presented with nausea and vomiting. He was found to be obstructed and required placement of a colonic stent. He was initiated on systemic chemotherapy but developed a large bowel obstruction again. The patient was then brought to the operating room and underwent an extended right colectomy with en bloc resection of the lateral wall of D2. The defect in the duodenum was reconstructed using a retrocolic Roux-en-Y D-J. The patient had an unremarkable initial hospital course but was re-admitted to the hospital for drainage of a peri-hepatic abscess and a superficial wound infection. Six months after the initial operation, the patient underwent partial hepatectomy of segments 3 and 8 also complicated by development of a liver abscess requiring drainage. The duodenal margin on his first operation was negative and he is currently alive with disease ( 22 months from surgery). He suffered a distant recurrence of disease.

\section{Patient 3}

A 61-year-old man symptomatic with abdominal bloating and discomfort was diagnosed with a duodenal adenoma on esophago-gastroduodenoscopic examination in D2/D3 which was one third the circumference of the duodenum $4 \mathrm{~cm}$ distal to the ampulla and unresectable endoscopically. The patient was brought to the operating room and after performing the Kocher maneuver the mass was found to be located in the duodenum as it swept under the mesenteric vessels. It felt distant from the ampulla and was segmentally resected confirming the diagnosis of duodenal tubular adenoma with negative margins and no invasive cancer. The duodenal wall was reconstructed via a retrocolic Roux-en-Y D-J with a $70 \mathrm{~cm}$ Roux limb. The patient's hospital course was uncomplicated and the patient was followed for ten months without evidence of

Table 1. Patient demographic data.

\begin{tabular}{|c|c|c|c|c|c|c|c|}
\hline Patient & Age (years) & Gender & BMI & Preoperative albumin & ASA classification & Co-morbidities & $\begin{array}{l}\text { Neoadjuvant } \\
\text { chemotherapy? }\end{array}$ \\
\hline 1 & 67 & M & 26.9 & 4 & 3 & DM, HTN, CAD & No \\
\hline 2 & 49 & M & 23.5 & 3.4 & 2 & HTN & Yes \\
\hline 3 & 61 & M & 30.4 & 4.1 & 2 & None & No \\
\hline 4 & 63 & $\mathrm{~F}$ & 27.7 & 2.8 & 3 & HTN & No \\
\hline 5 & 70 & $\mathrm{~F}$ & 31.3 & 4.2 & 3 & COPD, hypothyroidism & No \\
\hline
\end{tabular}

BMI, body mass index; ASA, American Society of Anesthesiologists; DM, diabetes mellitus; HTN, hypertension; CAD, coronary artery disease; COPD, chronic obstructive pulmonary disease.

Table 2. Patient operative data and peri-operative outcomes.

\begin{tabular}{|c|c|c|c|c|c|c|}
\hline Patient & $\begin{array}{l}\text { Extrinsic/ } \\
\text { intrinsic }\end{array}$ & $\begin{array}{l}\text { Tumor } \\
\text { location }\end{array}$ & $\begin{array}{l}\text { Type of duodenal } \\
\text { resection }\end{array}$ & EBL (mL) & LOS (days) & $\begin{array}{c}\text { Complications } \\
\text { (Clavien-Dindo score) }\end{array}$ \\
\hline 1 & Intrinsic & Infra-ampullary & Lateral duodenectomy & 300 & 20 & $\begin{array}{l}\text { Bile leak from T-tube (I), } \\
\text { peri-hepatic abscess (IIIa) }\end{array}$ \\
\hline 2 & Extrinsic & Infra-ampullary & Lateral duodenectomy & 500 & 8 & $\begin{array}{l}\text { Peri-hepatic abscess (IIIa), } \\
\text { superficial wound infection (I) }\end{array}$ \\
\hline 3 & Intrinsic & Infra-ampullary & Segmental duodenectomy & 400 & 6 & None \\
\hline 4 & Extrinsic & Infra-ampullary & Lateral duodenectomy & 700 & 22 & $\begin{array}{l}\text { Sepsis (IV), intra-abdominal } \\
\text { abscess (IIIa) }\end{array}$ \\
\hline 5 & Intrinsic & Infra-ampullary & Lateral duodenectomy & 450 & 11 & $\begin{array}{l}\text { Re-operation for gastrointestinal } \\
\text { bleed (IIIb) }\end{array}$ \\
\hline
\end{tabular}

EBL, estimated blood loss; LOS, length of stay. 
further disease.

\section{Patient 4}

A 63-year-old woman who presented with worsening dyspnea and light headedness. She was found to be severely anemic and was found to have a 10-12 cm circumferential ulcerating and necrotic mass in the ascending colon extending into the duodenum (D2), gallbladder and Segment $V$ of the liver. The patient underwent a Segment $V$ liver resection, cholecystectomy, right hemicolectomy and resection of the lateral duodenal wall invaded by tumor to grossly clear margins and reconstruction with retrocolic Roux-en-Y D-J. Pathology demonstrated a $14 \mathrm{~cm}$ tumor lymphovascular invasion, nine of ten positive lymph nodes and metastasis to para-aortic lymph nodes. Her hospital course was complicated by ureteral obstruction, aspiration, sepsis and intraabdominal abscess treated with percutaneous drainage. The patient is currently alive with disease ( 22 months post-operatively) with subsequent post-operative computerized tomography scans demonstrating nodular intra-peritoneal and retro-peritoneal tumor implants.

\section{Patient 5}

A 70-year-old woman presented with midabdominal pain and on computerized axial tomography scan was found to have a mass abutting the pancreatic head and D2. On endoscopic ultrasound the patient was seen to have a $2 \times 3 \mathrm{~cm}$ oval mass in the region of the uncinate process of the pancreas and appeared to be rising from the duodenal wall. A biopsy of this mass revealed spindle cells. Intra-operatively the mass was found to be $2 \mathrm{~cm}$ from the ampulla in a lateral inferior location. The ampulla was identified and preserved using a Fogarty catheter threaded through the cystic duct. After making a longitudinal duodenotomy the mass was resected via partial duodenectomy followed by retrocolic Roux-en-Y duodenojejunal reconstruction. On post-operative day one the patient was found to have a drop in hematocrit and bloody nasogastric tube output and was taken back to the operating room. Ontable upper endoscopy was performed and no bleeding was noted at the D-J, the jejunojejunostomy was opened and inspected with evacuation of old clot and no source of active bleed. The patient was presumed to have suf- fered a gastric bleed. The patient's hospital course thereafter was unremarkable. The radial margin of this resection was positive as the tumor extended beyond the wall of the duodenum and into the pancreas. The lateral margins were negative. She is alive without disease at 6 months from surgery.

\section{Overall operative data}

Patient operative data and perioperative outcomes are summarized in Table 2. The mean operative time for this series was 330 minutes (range 194-507 minutes). Mean EBL was $470 \mathrm{~mL}$ (range $300-700 \mathrm{~mL}$ ). The overall complication rate in these five patients was $80 \%$ with 7 total complications $-28.6 \%$ minor (Clavien-Dindo 1-2), and $71.4 \%$ major (Clavien-Dindo 3-5). Most post-operative complications were infectious and included three patients with intra-abdominal abscesses and one patient with a superficial wound infection. One patient was taken back to the operating room for a gastrointestinal bleed and another experienced a bile leak from around a T-tube. There were no complications related to injury to the ampulla of Vater, no anastomotic leaks and no mortalities in the post-operative period. Length of stay was a median of eleven days (range 6-22 days). The patient who stayed twenty-two days experienced a course in the intensive care unit (ICU) with hemodynamic instability on vasopressors for sepsis secondary to aspiration, eventually recovering (Table 2). Patients were followed for a median of 17 months (range 6-86 months). Pathologic and oncologic outcomes are listed in Table 3. Of these patients none had local duodenal recurrences and those with metastatic cancer had distant disease progression.

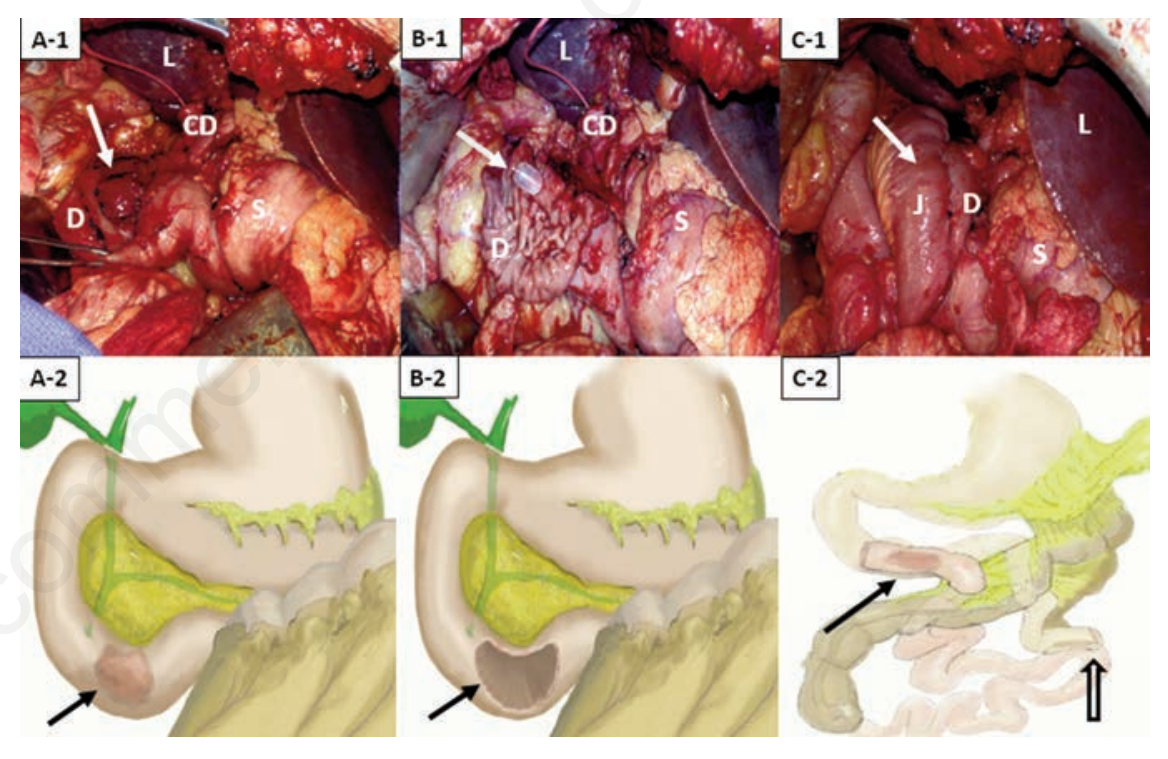

Figure 1. Operative technique used. A1) Intra-operative photograph of exophytic gastrointestinal stromal tumor from the posterior aspect of D2 (demonstrated by white arrow); A2) schematic of intrinsic D2 mass (black arrow); B1) post-resection duodenum with white arrow pointing to Fogarty balloon within ampulla of Vater; B2) black arrow shows partial duodenectomy of antimesenteric D2; C1) photograph of duodenojejunostomy (white arrow); C2) schematic of Roux-en-Y reconstruction [duodenojejunostomy

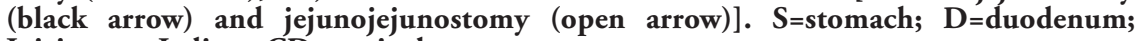
$\mathrm{J}=$ jejunum; $\mathrm{L}=$ liver; $\mathrm{CD}=$ cystic duct.

Table 3. Pathologic and oncologic data.

\begin{tabular}{|c|c|c|c|c|}
\hline Patient & Pathologic diagnosis & Margins & Local recurrence? & Disease status \\
\hline 1 & Moderately differentiated duodenal AC & Negative & No & NED \\
\hline 2 & Metastatic moderately differentiated colonic AC & Negative & No, new hepatic and pulmonary nodules & AWD \\
\hline 3 & Duodenal tubular adenoma & Negative & No & NED \\
\hline 4 & High grade poorly differentiated mucinous colonic AC & Positive & No, stable nodular implants & AWD \\
\hline$\underline{5}$ & \multicolumn{2}{|c|}{ Positive (radial margin) } & No & NED \\
\hline
\end{tabular}

AC, adenocarcinoma; NED, no evidence of disease; AWD, alive with disease; GIST, gastrointestinal stromal tumor. 


\section{Discussion}

In this study we analyzed cases of patients that underwent partial resection of the duodenum in a variety of locations that were subsequently reconstructed using a Roux-en-Y D-J. These patients had disparate pathologic diagnoses and underwent this operation for tumors both intrinsic and extrinsic to the duodenum. Up to now this method of reconstruction has only been described in case reports. Our series is the first to include data on operative and oncologic outcomes of patients who underwent duodenal resection for invasive cancer as well as primary duodenal tumors with subsequent reconstruction via Roux-en-Y D-J. In the literature this operation has been performed in majority for intrinsic duodenal tumors; mostly benign adenomas and GIST with good outcomes and few complications. ${ }^{4,5}$ We broadened the scope of this operation by including patients with high grade, invasive and even metastatic colon cancers that were causing obstructive symptomatology or bleeding as well as a case of primary duodenal adenocarcinoma. The high rate of complications in this small series of 5 patients is due to several factors including malnutrition ( $2 / 5$ patients with an albumin $<3.7$ with one patient at 2.8 ), as well as advanced cancer stage (stage IV) requiring neoadjuvant chemotherapy and multi-visceral organ resection. All of these factors are associated with higher infectious complications such as those experienced by patients 1, 2 and 4. Avoiding PD in these patients is preferred as the additional high morbidity associated with PD, in particular pancreatic fistula, in these patients would have been poorly tolerated.

Partial resection of the duodenum with Roux-en-Y reconstruction of the duodenal wall using a jejunal loop has been shown, in several case reports, to have utility in resection of tumors such as duodenal GIST that become large in size but do not metastasize via lymphatics or in the resection of benign duodenal adenomas. ${ }^{3,4}$ For these tumors, partial resection of the duodenum with reconstruction has equal oncologic outcomes to PD without the associated morbidity and mortality as long as clear margins are achieved..$^{5-8}$

Stauffer and colleagues who examined their institution's experience with laparoscopic partial duodenectomy of both benign disease as well as small adenocarcinomas with loop duodeno-jejunal reconstruction also found few complications and no recurrences at 11 months of follow up. ${ }^{8}$ We elected to perform one partial duodenectomy of a primary duodenal adenocarcinoma in D2 which was small and achieved negative margins on frozen sec- tion intra-operatively. In their analysis of duodenal adenocarcinoma patients, Bakaeen and colleagues showed that nodal metastases, positive margins and advanced stage of tumor was associated with decreased survival. However no difference in survival or ability to obtain negative pathologic margins was shown in patients who underwent PD vs partial duodenal resection. ${ }^{9}$

We found that in cases in which primary repair would compromise the duodenal lumen (resection of more than half the duodenal wall), Roux-en-Y reconstruction with a jejunal limb was safe and effective. Alternative methods of closing larger duodenal wall defects described in the literature include employing a jejunal serosal patch, a pedicle flap of terminal ileum and creation of a rectus abdominis musculo-peritoneal flap to cover the duodenotomy. ${ }^{10-13}$

These methods have been investigated in few case reports and series in variable disease processes, thus making comparative conclusions as to the efficacy of each repair method difficult.

\section{Conclusions}

In summary, oncologic resections of D2 and D3 portions of the duodenum for benign and malignant tumors can be performed in certain circumstances sparing the patient the morbidity of a PD. In these circumstances reconstruction of the duodenum is preferred over primary repair when there is a large defect in the duodenal wall. Identification of the ampulla is a critical step in the procedure that can be aided by the placement of a trans-biliary catheter intraoperatively. Reconstruction with a Rouxen-Y D-J in this series had a high rate of infectious complications likely because of this operation being performed in symptomatic patients with advanced and aggressive gastrointestinal cancers. Overall, there were no ampullary injuries, anastomotic leaks or perioperative deaths indicating that this method of reconstruction is relatively safe and can provide acceptable gastrointestinal and oncologic outcomes with no evidence of local recurrence and distant metastases only in patients who had Stage IV cancer upon initial presentation.

\section{References}

1. Androulakis J, Colborn GL, Skandalakis PN, et al. Embryologic and anatomic basis of duodenal surgery. Surg Clin N Am 2000;80:171-99.
2. Wolfman EF Jr., Trevino G, Heaps DK, Zuidema GD. An operative technic for the management of acute and chronic lateral duodenal fistulas. Ann Surg 1964;159:5639.

3. Fujiwara H, Yamasaki M, Nakamura S, et al. Reconstruction of a large duodenal defect created by resection of a duodenal tubulovillous adenoma using a doubletract anastomosis to a retrocolic roux-en-y loop: report of a case. Surg Today 2002;32: 824-7.

4. Mouaqit 0, Chbani L, Maazaz K, et al. A large gastrointestinal stromal tumor of the duodenum treated by partial duodenectomy with Roux-en-Y duodenojejunostomy: a case report. Available from: http://www. jmedicalcasereports. com/content/7/1/184

5. Mennigen R, Wolters HH, Schulte B, Pelster FW. Segmental resection of the duodenum for gastrointestinal stromal tumor (GIST). World J Surg Oncol 2008;6: 105.

6. Chung JC, Kim HC, Chu CW. Segmental duodenectomy with duodenojejunostomy of gastrointestinal stromal tumor involving the duodenum. J Korean Surg Soc 2011;80:12-16.

7. Goh BK, Chow PK, Ong HS, Wong WK. Gastrointestinal stromal tumor involving the second and third portion of the duodenum: treatment by partial duodenectomy and Roux-en-Y duodenojejunostomy. J Surg Oncol 2005;91:273-5.

8. Stauffer JA, Raimondo M, Woodward TA, et al. Laparoscopic partial sleeve duodenectomy (PSD) for nonampullary duodenal neoplasms: avoiding a whipple by separating the duodenum from the pancreatic head. Pancreas 2013;42:461-6.

9. Bakaeen FG, Murr MM, Sarr MG, et al. What prognostic factors are important in duodenal adenocarcinoma? Arch Surg 2000;135:635-41.

10. Ishiguro S, Moriura S, Kobayashi I, et al. Pedicled ileal flap to repair large duodenal defect after right hemicolectomy for right colon cancer invading the duodenum. Surg Today 2004;34:386-8.

11. Biyani DK, Speake D, Siriwardena A, Watson AJ. Management of duodenal involvement in locally advanced colonic carcinoma. Colorectal Dis 2007;9:178-81.

12. Yuan L, Zhou J, Shu G, et al. Pedicled ileal flap for duodenal defect after right hemicolectomy. Hepatogastroenterology 2010;57:493-6.

13. Agarwal P, Sharma D. Repair of duodenal fistula with rectus abdominis musculoperitoneal (RAMP) flap. Indian J Gastroenterol 2004;23:143-4. 